\title{
Durable antibacterial finish on cotton fabric using hydrogel-silver nanocomposite
}

\author{
Hossein Hosseinzadeh* and Sina Mohammadi
}

Chemistry Department, Payame Noor University, 19395-4697, Tehran, Iran

\begin{tabular}{l}
\hline C H R O N I C L E \\
\hline Article history: \\
Received March 26, 2013 \\
Received in Revised form \\
June 27, 2013 \\
Accepted 28 June 2013 \\
Available online \\
2 July 2013 \\
\hline Keywords: \\
Nanocomposite \\
Hydrogel \\
Acrylic acid \\
Itaconic acid \\
Antibacterial \\
\end{tabular}

\section{A B S T R A C T}

\begin{abstract}
A new durable antibacterial cotton fabric was successfully prepared by free-radical graft copolymerization of acrylic acid (AA) and itaconic acid (IA) onto a cotton fabric in an aqueous medium. Ammonium persulfate (APS) was used as the initiator in the presence of a crosslinker, methylene bisacrylamide (MBA). The nanocomposite hydrogel was obtained from in situ formation of silver nanoparticles from reduction of silver cations by sodium borohydride. A proposed mechanism for nanocomposite formation was suggested and the effect of ratio of IA to AA on water absorbency discussed. FTIR, UV-Vis, X-ray, and scanning electron microscopy were employed to characterize the structure of the prepared superabsorbent. The antibacterial activity of the hydrogel was tested qualitatively and quantitatively. Results showed that the silver nanoparticle-loaded fabric has potent antibacterial activity to Escherichia coli Gramnegative bacteria.
\end{abstract}

\section{Introduction}

Textile industry has made significant advances in developing antibacterial fibers and agents. Today, textile materials are used widely in various environments and antimicrobial treatment is rapidly becoming a prerequisite for textile goods used in hospitals, hotels, sports, and personal care industries1. Common problem in hospitals and healthcare institutions is microbial contamination of surfaces, including textile fabrics, which can lead to infections and consequently to cross-infections. Therefore, it is essential to reduce the transmission of harmful microorganisms and the spread of the secondary infections within a curative environment. Hospital acquired infections are prolonging the healing of patients and also representing the extra costs to the health service.

Accordingly, it is extremely important that protective clothing and hospital linen meet the demands for antimicrobial protection ${ }^{2}$. In order to reduce of transmission of infectious agents by contaminated protective equipment like masks, an antimicrobial nanoparticle coating has been developed. These

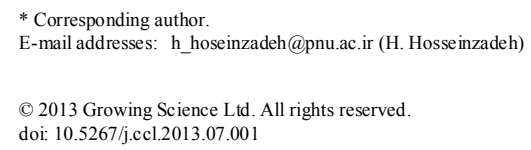


chemical agents were pulverized into particles less than $100 \mathrm{~nm}$ in size so as to increase their surface area, and to improve their microbicidal action ${ }^{3,4}$.

Noble metal nanoparticles have been of great scientific interest due to their unique properties at the nanoscale and wide range of applicability for optical, catalytic, electrical, magnetic and antimicrobial devices $^{5}$. Silver or silver ions are known for their powerful antibacterial activities ${ }^{6,7}$, which is generally believed to result from the reaction of this heavy metal with proteins. The chemical reaction of silver atoms with the OSH groups of enzymes inactivates the protein ${ }^{8}$. Silver has a broad antibacterial activity while exhibiting low toxicity towards mammalian cells ${ }^{9,10}$. Usually, carboxylic acid groups can be used as a temporary anchoring agent for attaching silver cations to polymer hydrogels, and the silver is reduced to form nanoparticle with borohydride salts ${ }^{11}$.

In this article, we report the results of our study using the graft co-polymerization to modify cotton fabric with the hydrogel-silver nanocomposite in order to impart the cotton with an antibacterial property. Silver nanoparticles add antibacterial properties to a variety of polymer hydrogels. This material can be washed several times without any loss of antibacterial activity and it increases the durability of fabric antibacterial activity.

\section{Results and Discussion}

\subsection{Mechanism of fabric-g-poly(AA-co-IA) nanocomposite formation}

The mechanism of grafting vinyl monomers onto cellulose using ammonium persulfate as oxidizing initiator is shown in Fig. 1. The sulfate anion-radicals produced from the thermal decomposition of APS abstract hydrogen from the hydroxyl groups of the polysaccharide substrate to form corresponding alkoxy radicals on the substrate. The alkoxy radicals in active centers on the substrate initiate the polymerization of AA and IA and lead to a graft copolymer ${ }^{12,13}$.

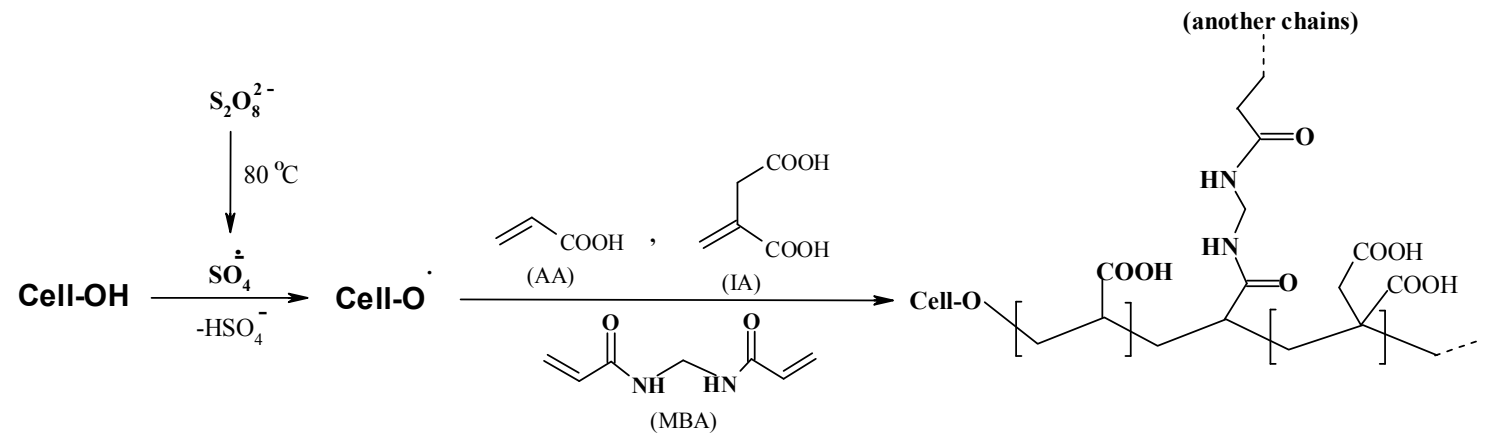

Fig. 1. Proposed mechanistic pathway for synthesis of fabric- $g$-poly(AA-co-IA)

The macromolecular gel network will serve effectively as nanoreactors for silver nanoparticle preparation. Fig. 2 depicts a scheme for the formation of silver nanoparticles within the swollen copolymeric network. When a fully swollen hydrogel is put in the aqueous $\mathrm{AgNO}_{3}$ solution, there occurs an ion exchange between $\mathrm{Ag}^{+}$ions present in the outer solution and $\mathrm{H}^{+}$ions present within the gel phase. After equilibrium is attained, the hydrogel is transferred into $\mathrm{KBH}_{4}$ solution. This results in a reduction of $\mathrm{Ag}^{+}$ions to silver nanoparticles within the swollen network. In this way, an almost uniformly distributed array of silver nanoparticles is obtained within the polymer network. The hydrogel turns slightly brown due to the presence of Ag nanoparticles. The most advanced features of this methodology are that the formed silver nanoparticles provided excellent stability over a longer period of time inside the hydrogel networks through the carboxylate groups of co polymeric chains ${ }^{14}$. 


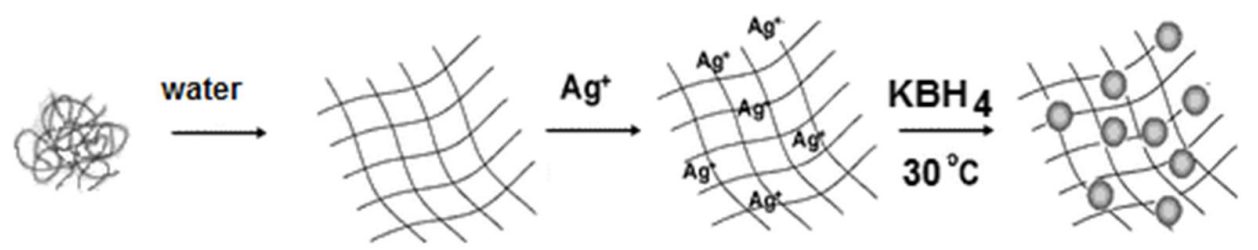

Fig. 2. Formation of silver nanoparticles

\subsection{Effect of Ratio of IA to AA on Water Absorbency}

In the poly(AA-co-IA) hydrogel, the water absorbency can be adjusted by changing the weight ratio of IA to AA with the weight of initial total monomers fixed. Fig. 3 presents that the equilibrium water absorbency of various samples increased as the mol ratio of IA to AA increased up to 0.08 . The incorporation of IA into the polymer network with higher IA content would lead to an increase in electrostatic repulsive force between charge sites on carboxylate ions upon their dissociation and enhance a more extended configuration, which might cause a higher swelling ratio of the hydrogels in tap water. But with higher increase in the ratio of IA in monomer composition, the water absorbency decreased because the presence of IA in the monomer mixture made the hydrogel formation more difficult. It is known that increasing the amount of IA in copolymeric structure decreases the effective crosslinking densities of polymer networks ${ }^{15}$.

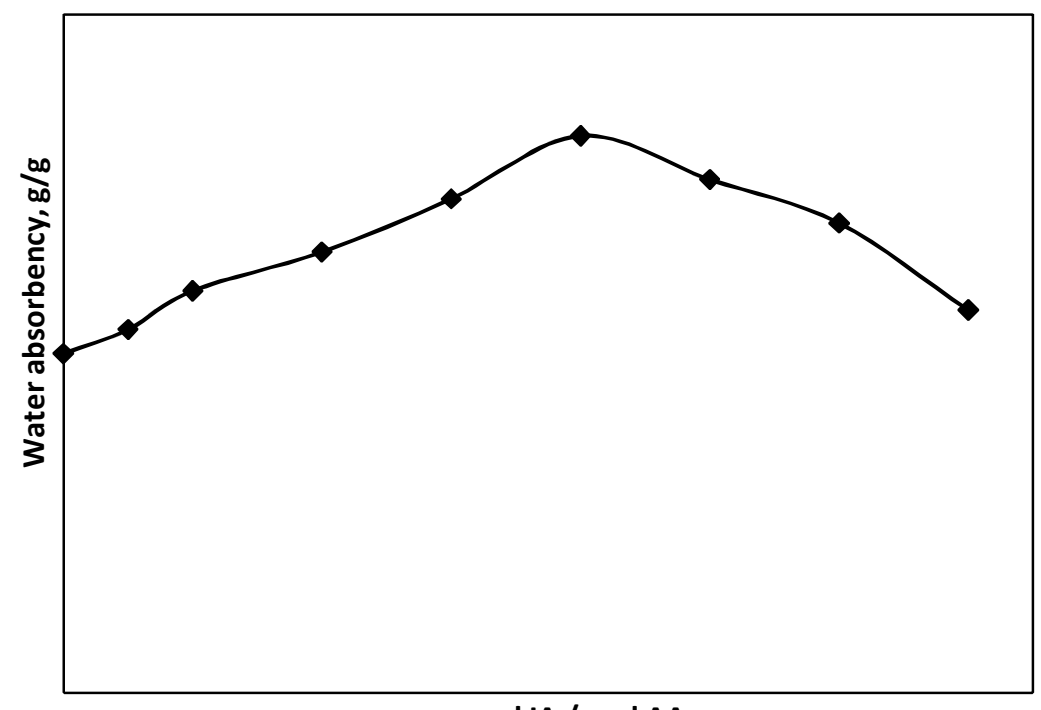

mol IA / mol AA

Fig. 3. Effect of IA/AA mol ratio on swelling capacity

\subsection{Spectral characterization}

\subsubsection{FTIR analysis}

A comparative depiction of FTIR spectra of cotton, poly (AA-co-IA)-grafted cotton, and Agloaded grafted cotton fabric is shown in Fig. 4. A broad peak corresponding to the $-\mathrm{OH}$ hydroxyl group of cotton fabric is observed in the $3200-3600 \mathrm{~cm}^{-1}$ range and a peak of the $-\mathrm{CO}$ carbonyl group is observed at $1651 \mathrm{~cm}^{-1}$. Asymmetric $\mathrm{C}-\mathrm{H}$ stretching is obtained in the $2000-2500 \mathrm{~cm}^{-1}$ range, whereas the symmetric peak is obtained in the $2600-2800 \mathrm{~cm}^{-1}$ range. A broad peak at 3400-3600 $\mathrm{cm}^{-1}$ in the spectrum of the grafted sample is due to the presence of both carboxylic groups of IA and AA. However, this broad peak almost disappears in the spectrum of Ag loaded fabric. In addition, the peak at $3282 \mathrm{~cm}^{-1}$, corresponding to carboxyl groups' functionalities in a grafted sample, is shifted to $3340 \mathrm{~cm}^{-1}$ in the spectrum of Ag-loaded fabric. This also indicates the binding of an Ag with oxygen in carboxyl groups ${ }^{16}$. 


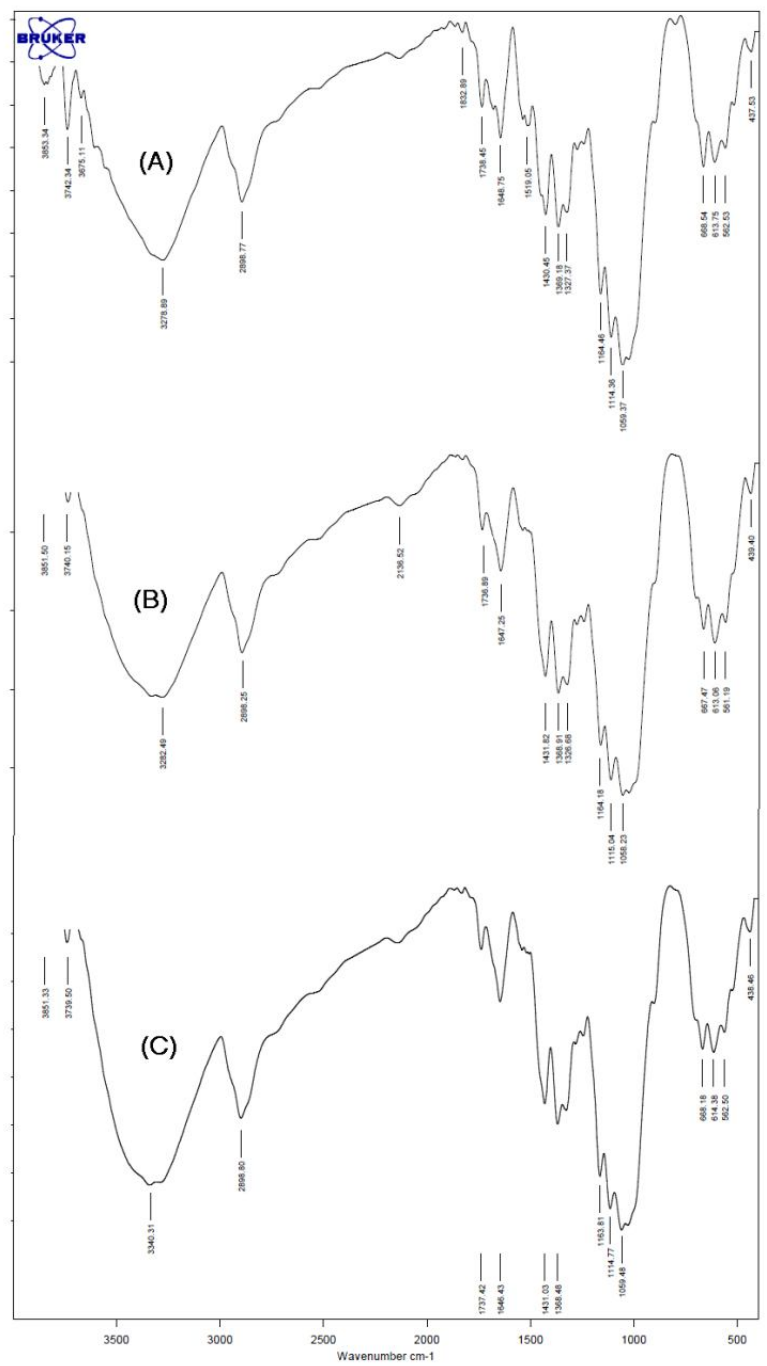

Fig. 4. FTIR spectra of cotton (A), cotton- $g$-poly(AA-co-IA) (B), and cotton- $g$-poly(AA-co-IA)/Ag nanoparticle $(\mathrm{C})$

\subsubsection{UV-visible analysis}

Further, to confirm the formation of silver nanoparticles in the hydrogel, we carried out UVvisible absorption studies. In Fig. 5, a strong characteristic absorption peak around $410 \mathrm{~nm}$ is noted for the silver nanoparticles ${ }^{16}$. However, the plain poly(AA-co-IA) hydrogel did not show any such peak.

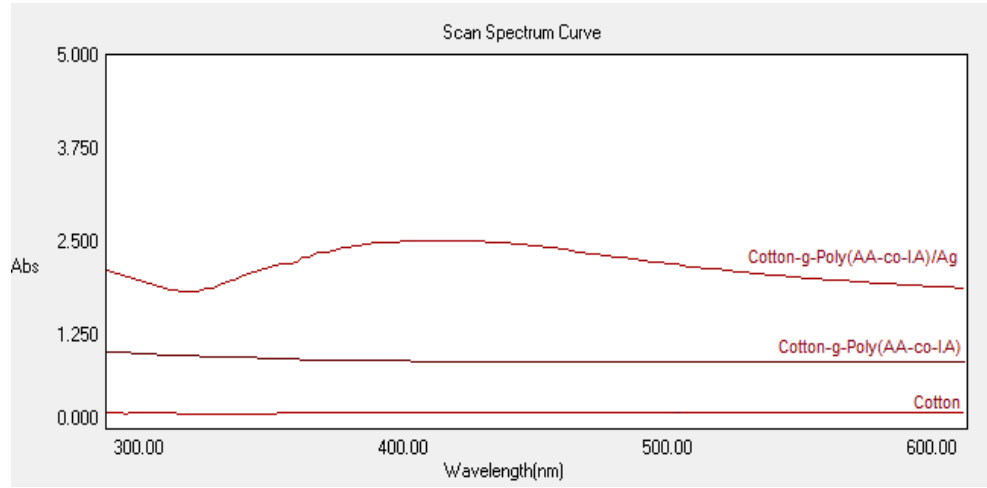

Fig. 5. UV-vis spectra of cotton, cotton- $g$-poly(AA-co-IA), and cotton- $g$-poly(AA-co-IA)/Ag nanoparticle 


\subsubsection{SEM analysis}

The scanning electron micrographs of cotton fabric and grafted cotton fabric are shown in Fig. 6. It can be seen that grafted cotton fabric has thin layer of poly(AA-co-IA) on surface. This phenomenon might be attributed to the hydrogen bonds between cotton fabric and grafted chains.

\subsubsection{XRD Analysis}

Structural information of silver nanoparticles was also obtained by X-ray diffraction (Fig. 7). The peak exhibited at $37.8^{\circ}$ is assigned to reflection through (111) plane of the face centered cubic (fcc) of silver nanoparticles, respectively. However, cotton-g-poly(AA-co-IA) did not show any such peak. Therefore, it is very clear that hydrogel nanocomposite consists of silver nanoparticles.

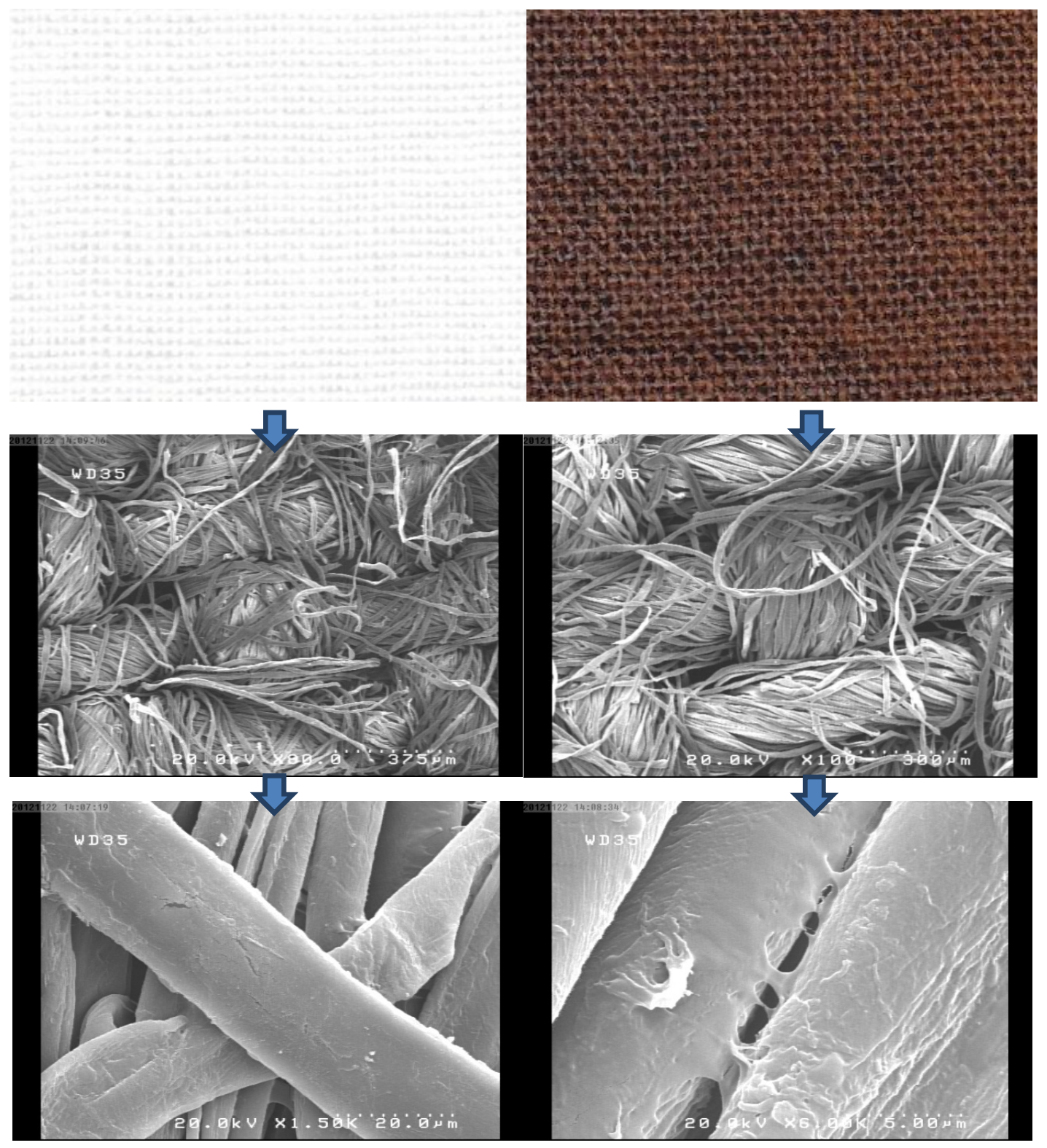

(A)

(B)

Fig. 6. SEM photograph of the cotton fabric (A) and the grafted cotton fabric (B) with various magnifications 


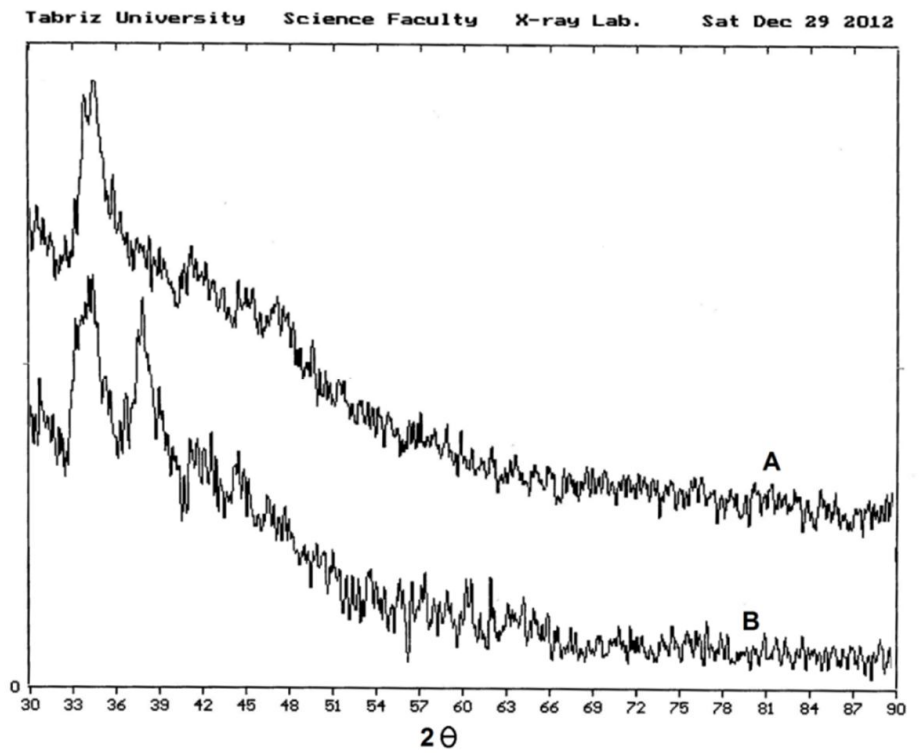

Fig. 7. XRD of cotton- $g$-poly(AA-co-IA) (A) and cotton- $g$-poly(AA-co-IA)/Ag nanoparticle

\subsection{Antibacterial activity of hydrogel nanocomposite}

\subsubsection{Disk diffusion method}

Antibacterial activity of fabric samples was determined in terms of inhibition zone formed on agar medium (Fig. 7). The control samples were normal cotton, which did not show any antibacterial activity (bacterial growth was seen on surface as indicated by the presence of colonies). The Agloaded cotton fabric placed on the bacteria-inoculated surfaces killed all the bacteria under and around them. We observed distinct zones of inhibition (clear areas with no bacterial growth) around the cotton samples for both E. coli. High bacterial growth as indicated by bacterial growth lawn (large indistinguishable collection of colonies) was observed everywhere. The observed zone of inhibition is a result of the leaching of active biocidal species $\mathrm{Ag}^{+}$ion from the embedded $\mathrm{Ag}$ particles present in the fabric ${ }^{17}$. The presence of the inhibition zone clearly indicates that the mechanism of the biocidal action of the fabric is due to the leached $\mathrm{Ag}^{+}$ion.

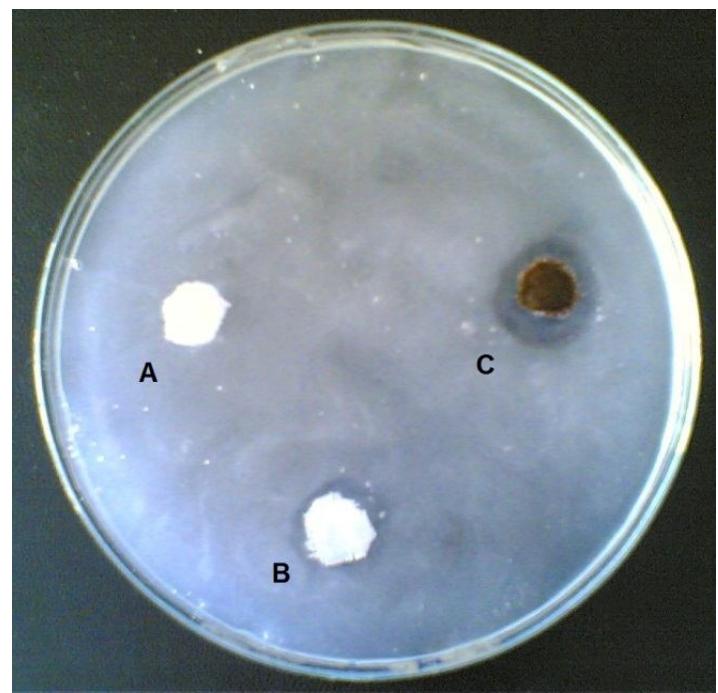

Fig. 7. Antibacterial activity of cotton (A), cotton- $g$-poly(AA-co-IA) (B), cotton- $g$-poly(AA-co-

$$
\mathrm{IA}) / \mathrm{Ag} \quad \text { nanoparticle (C) }
$$




\subsubsection{Colony forming units method}

Antibacterial activity of grafted and plan fabrics shows the antibacterial activity of E. coli for fabrics. The antibacterial activity was also determined by colony forming units (CFU) with time intervals (Table 1). The cotton fabric has no antibacterial effect, but grafted fabric exhibited at most 78\% reduction (R) for E. coli. The Ag-coated fabric showed 98\% reduction for E. coli.

Table 1. The reduction percentage $(\mathrm{R})$ of $\mathrm{CFU}$ with various time intervals

\begin{tabular}{lcc}
\hline Sample & \%R $(30 \mathrm{~min})$ & \%R $(60 \mathrm{~min})$ \\
\hline Control & 0 & 0 \\
Cotton & 3 & 3 \\
Cotton-g-Poly(AA-co-IA) & 56 & 78 \\
Cotton-g-Poly(AA-co-IA)/Ag & 86 & 97 \\
\hline
\end{tabular}

\subsubsection{Mechanism of antimicrobial activity of silver nanoparticles}

Several investigations have been carried out on the mechanism of bactericidal activity of silver nanoparticles. It is generally believed that heavy metals react with proteins by combining the $-\mathrm{SH}$ groups, which leads to the inactivation of the proteins. Recent research has demonstrated that the antimicrobial activities of silver nanoparticles depend on chemisorbed $\mathrm{Ag}^{+}$, formed on the surface of silver nanoparticles due to their extreme sensitivity to oxygen. However, the mechanism of the delivery of silver ions from silver nanoclusters to the bacteria needs further investigation. It was also proposed that silver ions released from silver nanoparticles can interact with phosphorous moieties in DNA, resulting in inactivation of DNA replication that leads to the inhibition of enzyme functions ${ }^{18}$.

\section{Conclusions}

Cotton fabric was grafted with a poly (acrylic acid-co-itaconic acid) via ammonium persulfate induced graft co-polymerization in an aqueous medium. The grafted cotton fabric was loaded with Ag nanoparticles by entrapment of $\mathrm{Ag}+$ ions into a grafted polymer network followed by borohydride reduction. The resulting dark brown color of the grafted fabric indicated the formation of $\mathrm{Ag}$ nanoparticles within the polymer network part of the grafted fabric. This preparation of Ag-coated fabric can be used as a protective clothing (surgical masks, caps, gowns, etc.) and hospital linen meeting the demands for antimicrobial protection. In addition, this process could be used for the fabrication of antibacterial clinical applications.

\section{Experimental}

\section{Materials}

Acrylic acid, itaconic acid, methylene bisacrylamide, ammonium persulfate and silver nitrate were obtained from Merck and sodium borohydride salts were obtained from Sigma-Aldrich company. Cotton fabric was obtained from a local textile mill.

\section{Preparation of the fabric}

The fabric was soaked in a detergent solution for $60 \mathrm{~min}$ and followed by extensive washing with tap water until free from any detergent. The clean fabric was then washed with distilled water, squeezed, and allowed to dry in an air oven at $60{ }^{\circ} \mathrm{C}$, and finally stored in a vacuum desiccator ready for use. 
The poly (AA-co-IA)-grafted fabric was prepared by carrying out free radical aqueous copolymerization of AA and IA using MBA as the crosslinker and APS as the initiator. In brief, in order to prepare a sample, the appropriate amount of fabric was placed in a test tube $1 \mathrm{~mL} \mathrm{AA,} 0.3 \mathrm{~g}$ IA and $\quad 0.005 \mathrm{~g}$ crosslinker MBA were dissolved in water and the final volume was made $10 \mathrm{~mL}$. Then, $0.1 \mathrm{~g}$ APS was dissolved and the whole reaction mixture was transferred in the test tube (internal diameter $1 \mathrm{~cm}$ ) and kept in a water bath at $60{ }^{\circ} \mathrm{C}$ for a period of $2 \mathrm{~h}$. When the grafting time was over, the grafted fabric was washed thoroughly with distilled water, and then extracted with hot water for $5 \mathrm{~h}$ in order to dissolve any homopolymer which may have adhered to the surface of the fabric. The fabric was equilibrated in the ethanol for a period of $24 \mathrm{~h}$ to remove the water. The grafted samples were then allowed to dry in an air oven at $60{ }^{\circ} \mathrm{C}$, until constant weight.

\section{Preparation of silver nanoparticle-loaded fabric}

A dry preweighed piece of grafted fabric was equilibrated in distilled water for $24 \mathrm{~h}$. Thereafter, the swollen fabric was put in an aqueous solution of Ag nitrate prepared by dissolving $20 \mathrm{mg}$ of $\mathrm{AgNO}_{3}$ in $30 \mathrm{~mL}$ of double-distilled water for $24 \mathrm{~h}$. Next the $\mathrm{Ag}$ ions present in the fabric were reduced to $\mathrm{Ag}$ nanoparticles by putting the fabric in $0.62 \mathrm{mM}$ potassium borohydride solution at 30 ${ }^{\circ} \mathrm{C}$ for $24 \mathrm{~h}$. Finally the fabric was rinsed with distilled water and put in a dust free chamber at $50{ }^{\circ} \mathrm{C}$ until it gained constant weight.

\section{Characterization}

The Fourier transformation infrared (FT-IR) spectra of plain and silver nanoparticle-loaded fabric samples were recorded on a FTIR spectrophotometer using $\mathrm{KBr}$. Absorption measurements were carried out on a UV-visible spectrophotometer for fabric solution in the wavelength range 300-600 $\mathrm{nm}$. The dissolution process of fabric was carried out according to the literature ${ }^{19}$. One $\mathrm{g}$ fabric was added into $25 \mathrm{~g}$ aqueous $7 \mathrm{wt} \% \mathrm{NaOH} / 12 \mathrm{wt} \%$ urea solution. Then, the solution was pre-cooled to $-12{ }^{\circ} \mathrm{C}$ and stirred for $5 \mathrm{~min}$. The nanocomposite structural and morphological variations were observed by using a scanning electron microscope (SEM).

\section{Microbial experimentation}

The antibacterial activity of the fabric was tested qualitatively and quantitatively by the disk diffusion method $^{10,11,20}$ and reduction of colony forming units $(\mathrm{CFU})^{16,21,22}$ of Escherichia coli (Gram-negative bacterium). For disk diffusion method, a mixture of nutrient broth and nutrient agar in $1 \mathrm{~L}$ distilled water at $\mathrm{pH} 7.2$ as well as the empty Petri plates were autoclaved. The agar medium was then cast into the Petri plates and cooled in laminar airflow. Approximately $10^{5}$ colony-forming units of each bacterium were inoculated on plates, and then each fabric samples was planted onto the agar plates. All the plates were incubated at $37{ }^{\circ} \mathrm{C}$ for $24 \mathrm{~h}$, following which the zone of inhibition was measured. For colony forming units method, the initial concentration of E. coli concentration is 6 $\times 10^{5} \mathrm{CFU} \mathrm{mL}^{-1}$. The fabric specimens were placed in a sterilized container and then $0.2 \mathrm{~mL}$ of the microorganism aqueous suspensions was dropped onto the surface of the specimens. The inoculated microorganisms in the fabric specimens were cultured at $37{ }^{\circ} \mathrm{C}$ for various times. After cultivation of microorganisms, to release test microorganisms from the fabric specimens, the fabric specimens were placed in $20 \mathrm{~mL}$ of neutralization solution and shaken vigorously. A microorganism suspension $(0.1$ $\mathrm{mL}$ ) was drawn and transferred to a nutrient agar plate and then it was cultured at $37{ }^{\circ} \mathrm{C}$ for $24 \mathrm{~h}$. The reduction $(\mathrm{R})$ percentage of $\mathrm{CFU}$ was calculated by the following:

$\mathrm{R}(\%)=\mathrm{C}-\mathrm{A} / \mathrm{C} \times 100$, 
where, $\mathrm{C}$ and $\mathrm{A}$ are the bacterial colonies of the cotton fabric and the silver nanoparticle-loaded cotton fabrics respectively.

\section{References}

1. Mao, J., Murphy, L. (2001) Durable freshness for textiles. AATCC Rev, 1, 28-31.

2. Vigo, T. L. (2001) Antimicrobial Polymers and Fibers: Retrospective and Prospective. In: Bioactive Fibres and Polymers. American Chemical Society. Washington DC, 175-200.

3. Galeano, B., Korff, E. and Nicholson, W. L. (2003) Inactivation of vegetative cells, but not spores, of Bacillus anthracis, B. cereus, and B. subtilis on stainless steel surfaces coated with an antimicrobial silver- and zinc-containing zeolite formulation. Appl. Environ. Microbiol. 69, 4329-4331.

4. Li, Y., Leung, P., Yao, L., Song, Q. W. and Newton, E. (2006) Antimicrobial effect of surgical masks coated with nanoparticles. J. Hosp. Infect., 62, 58-63.

5. Park, S. Y., Chung, J. W., Priestley, R. D. and Kwak, S. Y. (2012) Covalent assembly of metal nanoparticles on cellulose fabric and its antimicrobial activity, Cellulose 19, 21412151.

6. Mohan, Y. M., Premkumar, T., Lee, K. and Geckeler, K. E. (2006) Fabrication of silver nanoparticles in hydrogel networks. Macromol. Rapid Commun. 27, 1346-1354.

7. Mohan, Y. M., Lee, K., Premkumar, T. and Geckeler, K. E. (2007) Hydrogel networks as nanoreactors: a novel approach to silver nanoparticles for antibacterial applications. Polymer $48,158-164$.

8. Jeon, H. J., Yi, S. C. and Oh, S. G. (2003) Preparation and antibacterial effects of Ag-SiO 2 thin films by sol-gel method. Biomaterials, 24, 4921-4928.

9. Sambhy, V. MacBride, M. M., Peterson, B. R. and Sen, A. (2006) Silver bromide nanoparticle/polymer composites: dual action tunable antimicrobial materials. J. Am. Chem. Soc. $128,9798-9808$.

10. Khalil-Abad, M. Sh., and Yazdanshenas, M. E. (2010) Superhydrophobic antibacterial cotton textiles, J. Colloid Interface Sci. 351, 293-298.

11. Xiang, Y. and Chen, D. (2007) Preparation of a novel pH-responsive silver nanoparticle/poly(HEMA-PEGMA-MAA) composite hydrogel. Eur. Polym. J. 43, 41784187.

12. Ni, B.L., Liu, M.Z., Lu, S.Y., Xie, L.H., Zhang, X., and Wang, Y.F. (2010) Novel slowrelease multielement compound fertilizer with hydroscopicity and moisture preservation. Ind. Eng. Chem. Res., 10, 4546-4552.

13. Wang, Y., Liu, M., Ni, B., Xie, L. and Zhang, X. (2011) Preparation and Properties of Novel Slow-release PK Agrochemical Formulations Based on Carboxymethylcellulose-GraftPoly(acrylic acid-co-itaconic acid) Superabsorbents. J. Macromol. Sci., Part A: Pure and Appl. Chem., 48, 806-815.

14. Thomas, V., Yallapu, M. M., Sreedhar, B. and Bajpai, S. K. (2007) A versatile strategy to fabricate hydrogel-silver nanocomposites and investigation of their antimicrobial activity. $J$. Colloid Interface Sci. 315, 389-395.

15. Sadeghi, M. and Ghasemi, N. (2012) Synthesis and study on effect of various chemical conditions on the swelling property of collagen-g-poly(AA-co-IA) superabsorbent hydrogel. Ind. J. Sci. Tech. 5, 1879-1884.

16. Selvam, S., Gandhi, R. R., Suresh, J., Gowri, S., Ravikumar, S. and Sundrarajan, M. (2012) Antibacterial effect of novel synthesized sulfated $\beta$-cyclodextrin crosslinked cotton fabric and its improved antibacterial activities with $\mathrm{ZnO}, \mathrm{TiO}_{2}$ and $\mathrm{Ag}$ nanoparticles coating. Inter. J. Pharm. 434, 366-374.

17. Wang, H., Wang, J., Hong, J., Wei, Q., Gao, W., Zhu, Z. (2007) Preparation and characterization of silver nanocomposite textile. J. Coat. Technol. Res. 4, 101-106. 
18. El-Rafie, M. H., Mohamed, A. A., Shaheen, Th. I. and Hebeish. A. (2010) Antimicrobial effect of silver nanoparticles produced by fungal process on cotton fabrics. Carbohyd. Polym. 80, 779-782.

19. Cai, J., Zhang, L., Liu, S. L., Liu, Y. T., Xu, X. J., Chen, X. M., et al. (2008) Dynamic selfassembly induced rapid dissolution of cellulose at low temperatures. Macromolecules, 41, 9345-9351.

20. Khalil-Abad, M. Sh., Yazdanshenas, M. E. and Nateghi, M. R. (2009) Effect of cationization on adsorption of silver nanoparticles on cotton surfaces and its antibacterial activity. Cellulose $16,1147-1157$.

21. Cao, Z., and Sun, Y. (2009) Polymeric N-halamine latex emulsions for use in antimicrobial paints. Appl. Mater. Interfaces 1, 494-504.

22. Li, Y., Leung, P., Yao, L., Song, Q.W. and Newton, E. (2006) Antimicrobial effect of surgical masks coated with nanoparticles. J. Hosp. Infect. 62, 58-63. 